Conf- $940933--24$

GA-A21834

\title{
EXPERIMENTAL CONSTRAINTS ON TRANSPORT
}

\author{
by \\ T.C. LUCE, C.C. PETTY, K.H. BURRELL, \\ C.B. FOREST, P. GOHIL, R.J. GROEBNER, \\ J.C.M. de HAAS, R.A. JAMES, \\ and M.A. MAKOWSKI
}

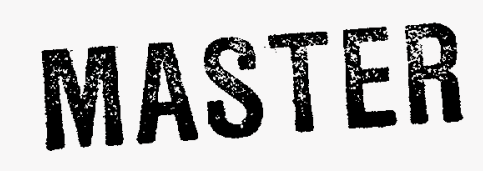

DISTRIBUTION OF THIS DOCUMENT IS UNLIMITED

DECEMBER 1994 


\title{
EXPERIMENTAL CONSTRAINTS ON TRANSPORT
}

\author{
by \\ T.C. LUCE, C.C. PETTY, K.H. BURRELL, \\ C.B. FOREST, P. GOHIL, R.J. GROEBNER, \\ J.C.M. de HAAS,* R.A. JAMES,* \\ and M.A. MAKOWSKI*
}

This is a preprint of a paper presented at the 15th International Conference on Plasma Physics and Controlled Nuclear Fusion Research, September 26-October 1, 1994, Madrid, Spain, and to be printed in the Proceedings.

\author{
Work supported by \\ U.S. Department of Energy Contracts \\ DE-AC03-89ER51114 and W-7405-ENG-48
}

* Lawrence Livermore National Laboratory

\section{GENERAL ATOMICS PROJECT 3466 DECEMBER 1994}

DISTRIBUTION OF THIS DOCUMENT IS UNLIMITED 


\section{DISCLAIMER}

This report was prepared as an account of work sponsored by an agency of the United States Government. Neither the United States Government nor any agency thereof, nor any of their employees, make any warranty, express or implied, or assumes any legal liability or responsibility for the accuracy, completeness, or usefulness of any information, apparatus, product, or process disclosed, or represents that its use would not infringe privately owned rights. Reference herein to any specific commercial product, process, or service by trade name, trademark, manufacturer, or otherwise does not necessarily constitute or imply its endorsement, recommendation, or favoring by the United States Government or any agency thereof. The views and opinions of authors expressed herein do not necessarily state or reflect those of the United States Government or any agency thereof. 


\section{DISCLAIMER}

Portions of this document may be illegible in electronic image products. Images are produced from the best available original document. 


\section{EXPERIMENTAL CONSTRAINTS ON TRANSPORT*}

T. C. Luce, C.C. Petty, K.H. Burrell, C.B. Forest, P. Gohil, R.J. Groebner, J.C.M. De HAAS ${ }^{\dagger}$ R.A. JAMEs, ${ }^{\dagger}$ and M.A. MaKowsKI ${ }^{\dagger}$

General Atomics, San Diego, California, United States of America

*This is a report of work supported by the U.S. Department of Energy under Contract Nos. DE-AC03-89ER51114 and W-7405-ENG-48.

$\dagger$ Permanent address: Lawrence Livermore National Laboratory, Livermore, California, U.S.A. 
IAEA-CN-60/A-2-III-2

\begin{abstract}
Characterization of the cross-field energy transport in magnetic confinement experiments in a manner applicable to the accurate assessment of future machine performance continues to be a challenging goal. Experimental results from the DIII-D tokamak in the areas of dimensionless scaling and non-diffusive transport represent progress toward this goal. Dimensionless scaling shows how beneficial the increase in machine size and magnetic field is for future devices. The experiments on DIII-D are the first to determine separately the electron and ion scaling with normalized gyroradius $\rho_{*}$; the electrons scale as expected from gyro-Bohm class theories, while the ions scale consistent with the Goldston empirical scaling. This result predicts an increase in transport relative to Bohm diffusion as $\rho_{*}$ decreases in future devices. The existence of distinct $\rho_{*}$ scalings for ions and electrons cautions against a physical interpretation of one-fluid or global analysis. The second class of experiments reported here are the first to demonstrate the existence of nondiffusive energy transport. Electron cyclotron heating was applied at the half radius; the electron temperature profile remains substantially peaked. Power balance analysis indicates that heat must flow in the direction of increasing temperature, which is inconsistent with purely diffusive transport. The dynamics of electron temperature perturbations indicate the presence in the heat flux of a term dependent on temperature rather than its gradient. These two observations strongly constrain the types of models which can be applied to cross-field heat transport.
\end{abstract}

\title{
1. INTRODUCTION
}

A fundamental obstacle in the design of a magnetic confinement fusion reactor is the lack of first-principles knowledge of the processes which govern cross-field particle and energy transport. The projected cost of such reactors depends sensitively on size [1], which makes inclusion of added performance margin by increasing the size an expensive design choice. The intuitive notion that larger devices have better confinement is validated by multiple machine databases, but the various "scaling laws" do not provide a unified answer for size scaling [2]. Therefore, the designer is not even sure how much margin he has. Furthermore, critical issues such as fusion yield and stability of the magnetohydrodynamic (MHD) instabilities depend sensitively on the plasma pressure profiles, not just the total stored energy, and prediction of the pressure profile requires knowledge of both the sources and the transport. It seems unlikely that a first-principles prediction of transport will be achieved in the course of the design of the next-step devices such as ITER; however, there is hope that the transport in future machines can be accurately predicted using dimensionless scaling. The first experiments to exploit the dimensionless scaling technique to determine independently the size scaling of electron and ion energy transport are reported in Section 2. 
Progress toward a first-principles description of cross-field plasma transport has been slowed due to the difficulty in evaluating the nonlinear turbulence which is the suspected cause of the transport, and the difficulty of accurately testing various models experimentally. Energy transport is quantified experimentally using a power balance analysis. This technique suffers in accuracy because it requires radial derivatives of relatively sparse experimental data. A more fundamental limitation, however, is that the transport must be characterized by a single parameter at each radius. The standard model is a linear force-flux relation with the heat conductivity as the unknown. Experiments which show this model to be inadequate to completely describe energy transport will be discussed in Section 3. Since this model is also invoked in the dimensionless scaling arguments, the implications of non-diffusive transport on the size scaling projections will be given in Section 4 .

\section{DIMENSIONLESS SCALING STUDIES}

The use of dimensionless variables is a standard practice in theoretical physics. A classical example is the description of fluid dynamics by the Navier-Stokes equation. The behavior of fluid can be predicted by knowing the value of a single dimensionless number known as the Reynolds number. The behavior of plasmas can be considerably more complex. Various approximations to the Vlasov-Maxwell system of equations used to describe plasma dynamics yield sets of dimensionless quantities which characterize the plasma $[3,4]$. Experimental studies of confinement and transport have focussed on the variation of engineering parameters such as density and current over which the experimenter has direct control rather than dimensionless quantities relevant to theories. This approach has been quite successful in finding the principal scalings of confinement, e.g., linear scaling with current $I$ and degradation of confinement with the square root of the input power $P$. These dependencies are validated by their presence on tokamaks of all types. One critical dependence that this approach does not determine well is the benefit of increasing machine size. Because this scaling cannot be done on a single machine, multiple machine data sets must be used. This, of course, introduces systematic errors and biases resulting in a wide variation in predicted size scaling [2]. It is exactly this scaling, however, which is best determined by the dimensionless scaling approach. Both neoclassical and quasilinear drift wave theory find a diffusivity of the form

$$
\chi=\chi_{\mathrm{B}}\left(\rho_{*}\right)^{\alpha} F\left(\beta, \nu_{*}, q, R / a, \kappa, \ldots\right),
$$

where $F$ is an arbitrary function of all of the relevant dimensionless parameters except $\rho_{*}$, the gyroradius normalized to the machine size. The diffusivity is normalized to $\chi_{\mathrm{B}} \equiv c T / e B$, the Bohm diffusion form, for convenience. Present day experiments can operate at values predicted for reactor operation for all dimensionless quantities except $\rho_{*}$. Therefore, the value of $F$ can be determined without knowing the complex functional dependence of each dimensionless 
quantity. The exponent $\alpha$ is then determined by varying $\rho_{*}$ while holding $F$ constant. The universality of $\alpha$ can be tested in much the same fashion as the universality of the current and input power scalings have been validated. That the knowledge of $\alpha$ determines the minimum reactor size can be demonstrated by the following argument. The optimum reactor operating temperature is determined by the fusion cross-section. The magnetic field strength is limited by technological constraints. The smallest reactor along a path of constant $F$ will be the one at the optimum temperature $T$ and maximum field $B$. Knowing $F, T, B$, and $\alpha$, the only remaining unknown is the machine scale length in $\rho_{*}$. Evaluating this at the optimum $T$ and $B$ gives the smallest possible reactor for a given fusion yield. Thus dimensionless scaling has the potential to accurately predict the transport behavior of future devices with little explicit knowledge of the parametric dependencies.

Before presenting the DIII-D results, it is useful to discuss the theoretical expectations for the value of $\alpha$. All low-frequency drift-wave calculations yield a diffusivity with a linear $\rho_{*}$ dependence. Neoclassical theory also gives a linear $\rho_{*}$ dependence. Both theories have a characteristic diffusive step which scales with gyroradius. With the diffusivity normalized to Bohm as in Eq. (1), this linear dependence of $\rho_{*}$ is known as "gyro-reduced Bohm" scaling or simply "gyro-Bohm." Although there are no theories which give $\alpha=0$, there is some experimental evidence of this scaling. This scaling is known as Bohm scaling since the diffusivity takes the form of the Bohm diffusivity multiplied by a constant. If radial transport were due to parallel transport along radially wandering field lines, then $\alpha$ must equal -1 , to eliminate the $B$ dependence. The scaling will be called "stochastic," since it would arise if no magnetic surfaces existed. Finally, the empirical scaling of confinement as $I / P^{1 / 2}$ can be written in dimensionless variables as inversely proportional to $\beta q^{2}$. This transformation is not unique because the original formula is not dimensionally correct, but it is the simplest conversion to dimensionless variables. Along a path where $F$ is constant, $\beta q^{2}$ is also constant. This would imply $\alpha=-1 / 2$ to keep $\chi$ constant. This scaling will be called "Goldston" scaling after the first appearance of such an empirical relation [5].

The $\rho_{*}$ scaling has been determined experimentally in three different regimes on DIII-D - low density rf heated, high density if heated, and high density neutral beam heated. In each case, the discharge shape and size are held constant while varying $\rho_{*}$. Keeping $F$ fixed implies a fixed relationship of $I, T$, and density $n$ to $B$ in order to keep $\beta, \nu$, and $q$ fixed:

$$
n \propto B^{4 / 3}, \quad T \propto B^{2 / 3}, \quad I \propto B
$$

which implies that $\rho_{*} \propto B^{-2 / 3}$. The toroidal field is varied by a factor of two so that the resonant heating by the rf has the same deposition profile. This variation in $B$ leads to a scan of $\rho_{*}$ by a factor of 1.6. By repeating many discharges, a well-matched pair was found for each regime. Both the electron and ion parameters were matched concurrently in the same discharge pair. 
In these experiments, it was possible for the first time to determine the $\rho_{*}$ scaling independently for electrons and ions. Previous experiments assumed that the two species had the same scaling. In all three cases, the results were identical - the electrons scaled as gyro-Bohm, while the ions followed Goldston scaling. As an example, the neutral beam data are shown in Fig. 1(a). In addition to the data from the individual species the global confinement scaling and the effective one-fluid diffusivity are shown. Both are consistent with Bohm scaling. This points out a serious pitfall waiting for those who use one-fluid analysis. The only reason the global and one-fluid analysis give Bohm scaling is that the power is exhausted almost equally by the electron and ion fluids [see Fig. 1(b)] and Bohm scaling lies between the gyro-Bohm and Goldston scalings. The Bohm scaling is a completely spurious result and is not indicative of the underlying physics!

The existence of distinct $\rho_{*}$ scalings for ions and electrons provides a unifying explanation of previous results which appeared contradictory. A summary of the DIII-D data is given in Fig. 2. In all three regimes, the electrons exhibit gyro-Bohm scaling while the ion scaling is Goldston. The low density if data has a global scaling close to the electron scaling because the input goes exclusively to the electrons. And, since the coupling to the ions is small at low density, the plasma transports most of the energy out through the electron fluid. This is similar to the experiments reported previously on DIII-D [6] and the Wendelstein VII-AS results [7]. In both cases, the one-fluid analysis of the low density rf heated discharges indicated gyro-Bohm scaling. For the high density if case, the global scaling is Bohm since roughly equal amounts of power are exhausted by both fluids, even though the heating is directly to electrons. This is similar to the JET result [8] using fast wave minority heating, which predominantly heats electrons, at a density where the electrons and ions are strongly coupled. Finally, the neutral beam case is like the high density if case except now the beam directly deposits power in the ions and the exchange term is not as important. This is similar to the TFTR result [9] that showed Bohm scaling for the one-fluid diffusivity. While these heuristic arguments do not constitute a proof that other experiments would see the same electron and ion scalings found in DIII-D, the appearance of distinct scalings for the two species can provide a unified explanation of all previous results.

If the scalings observed for electrons and ions are valid in reactor regimes, the confinement picture becomes very pessimistic at the smaller $\rho_{*}$ characteristic of reactors. For a two-species plasma at equal temperatures, the effective diffusivity can be written

$$
\chi_{\mathrm{eff}}=\frac{1}{2} \chi_{\mathrm{B}} \rho_{*} F\left[1+\left(\frac{m_{\mathrm{i}}}{m_{\mathrm{e}}}\right)^{1 / 4} \frac{G}{F} \rho_{*}^{-3 / 2}\right],
$$




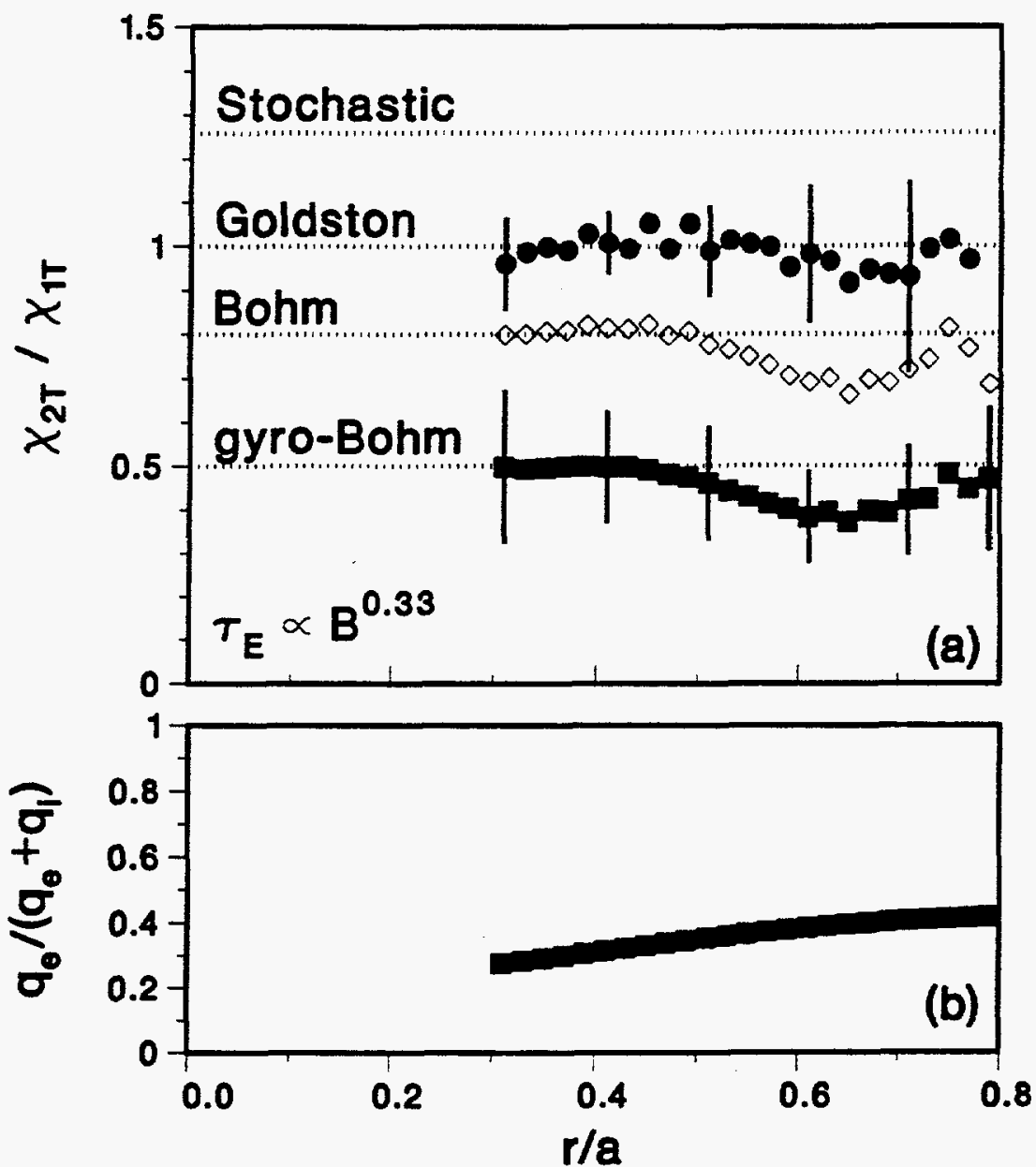

FIG. 1. (a) Plot of the ratios of electron (solid square), ion (solid circle), and effective (open diamond) power balance diffusivities at full field (2 T) and half field (1 T) versus normalized toroidal flux $\rho$. The discharges are heated by neutral beam injection at $q=5.8$ and $\beta_{\mathrm{N}}=0.5$. The electron ratio is consistent with gyro-Bohm scaling, while the ion ratio is consistent with Goldston empirical scaling (See text for the definitions of the labels.) Both the effective diffusivity and the global confinement exhibit Bohm scaling. (b) The fractional flux exhausted in the electron channel.

where $G$ is the dimensionless function analogous to $F$ for the ions. It is clear that $\chi_{\text {eff }}$ does not have the appropriate form assumed for diffusivity [Eq. (1)]. The $\rho_{*}$ dependence is illustrated in Fig. 3. For equal temperatures, the stronger transport scaling dominates. As $\rho_{*}$ decreases toward reactorrelevant values, the observed ion scaling dominates, and leads to more rapid transport. Because the highest performance machines of the present generation 


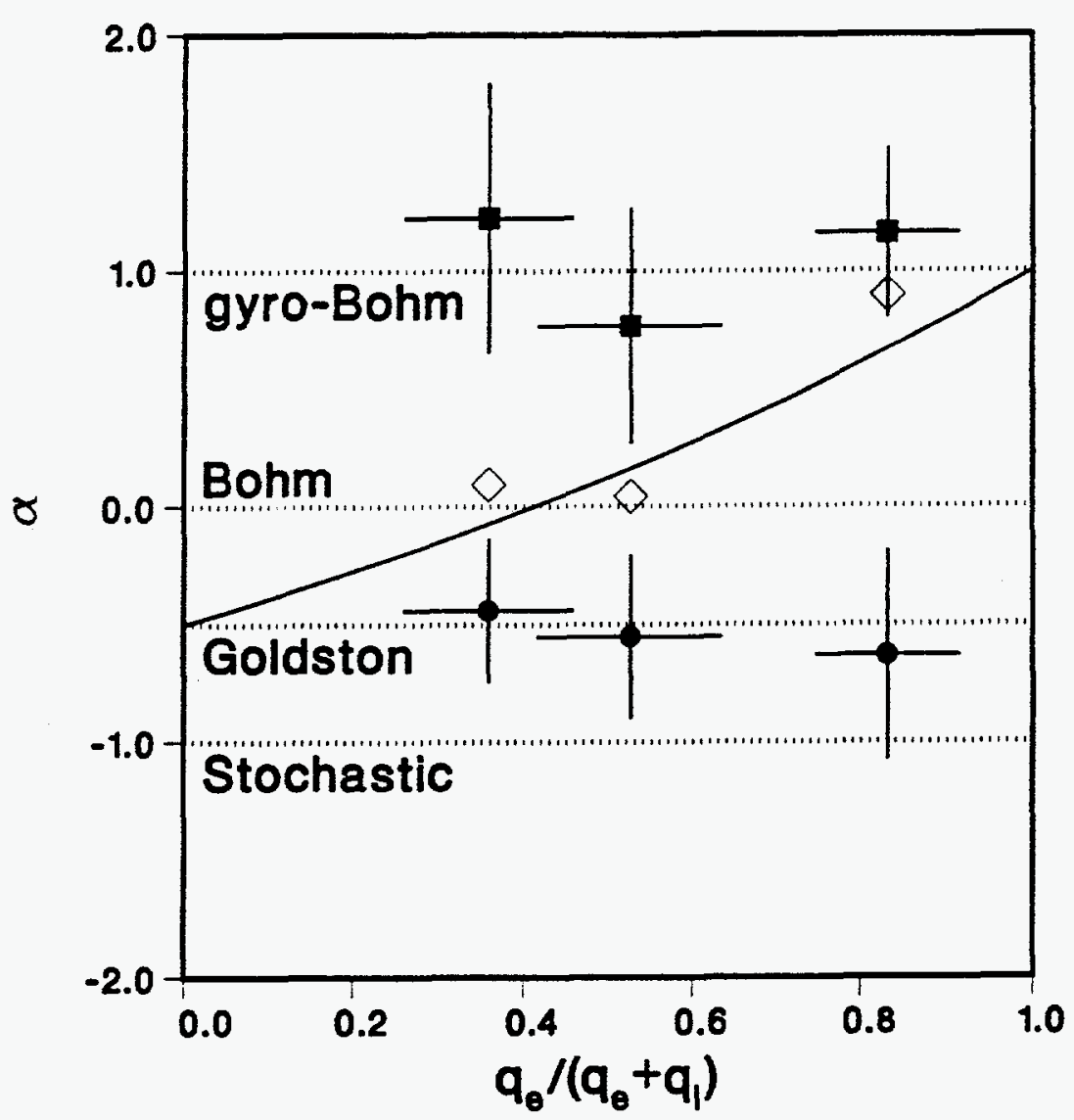

FIG. 2. The value of $\alpha$ plotted against the fraction of the total flux carried out by the electrons for the three cases discussed in the text. The symbols are the same as defined in Fig. 1. The left set of points is the NBI case from Fig. 1. The middle set is the high density of case and the right set is the low density if case. The solid line is the value of $\alpha$ which an effective diffusivity analysis would find for electrons with exactly gyro-Bohm scaling and ions with exactly Goldston scaling.

already observe Bohm scaling (which lies at the minimum of the curve), an unfavorable scaling of confinement with smaller $\rho_{*}$ is predicted. At the other extreme, for a neutral beam heated hot-ion mode discharge where all of the power is deposited and exhausted in the ion fluid, the observed scaling would predict that ion confinement would reach the neoclassical value at high $\rho_{*}$. Results from DIII-D [10] agree with this prediction. Because the ITER ignition design point does not lie on a path of constant $F$ (and $G$ ) from the present experiments, a definite prediction of ITER ignition performance cannot be 


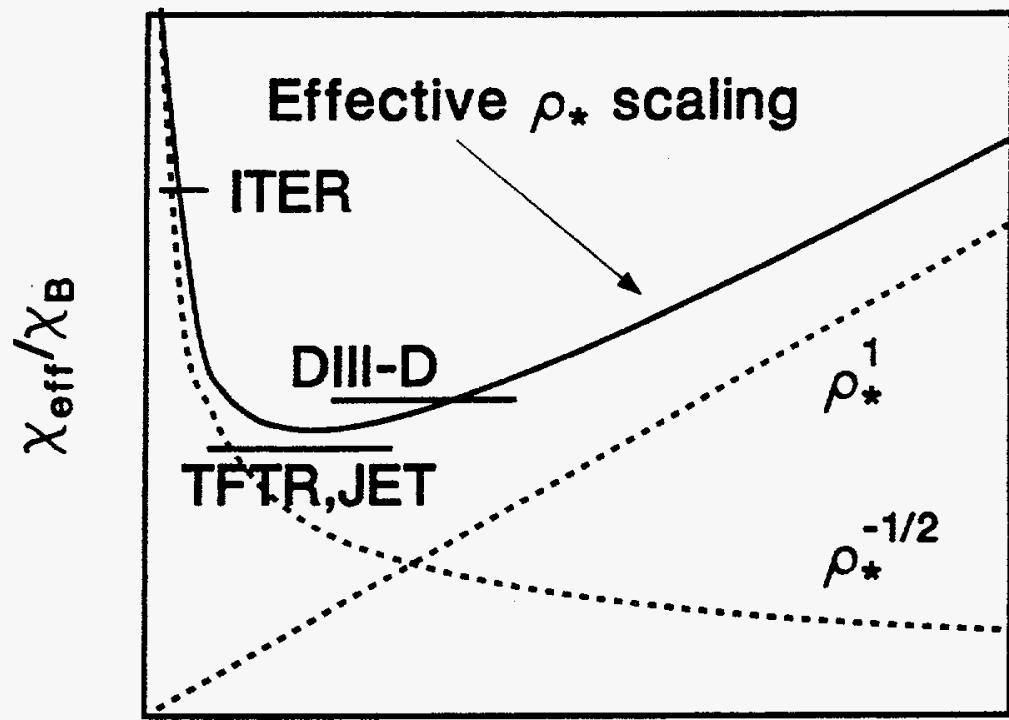

$\rho_{*}$

FIG. 3. The $\rho_{*}$ scaling of the effective diffusivity for the case $T_{\mathrm{e}}=T_{\mathrm{i}}$. The location of JET and TFTR experiments are specified by the reported results of Bohm scaling of the effective diffusivity. (Bohm scaling implies $\chi_{\mathrm{eff}} / \chi_{\mathrm{B}}$ is independent of $\rho_{*}$, which only occurs at the minimum of the graph.) ITER is projected to operate with $\rho_{*}$ approximately a factor of three smaller than JET. The ITER ignition design point does not lie on a $\rho_{*}$ scaling path from the $D I I I-D L$-mode results. The extrapolation is only valid along such a path in parameter space; therefore, an evaluation of ITER ignition performance is not strictly possible.

made on the basis of this data. Experiments which do lie on such a path are in progress.

\section{NON-DIFFUSIVE TRANSPORT}

As discussed above, most energy transport studies rely on power balance analysis which characterizes the transport by a single parameter, the heat diffusivity, at each radius. Most theories, however, take the form

$$
\vec{\Gamma}=\overrightarrow{\vec{M}} \vec{F}
$$


where the elements of $\Gamma$ are the fluxes of particles, momentum, and energy; $F$ is composed of the generalized driving forces, usually gradients of the densities, momenta, and energy. The matrix $M$ has diagonal elements which are the usual diffusivities, but the off-diagonal elements are non-zero, in general. The flux due to these terms will be called non-diffusive here because the total flux is not a simple linear force-flux relation. For particles, the flux has long been recognized as having a significant non-diffusive component [11]. The evidence for this is the existence of peaked profiles despite an edge-localized source. The electron temperature profiles have also been long recognized to respond much less to changes in the source than expected on the basis of a purely diffusive model. This observation is variously called "profile consistency" [12] or "profile resilience" [13]. A typical example is shown in Ref. 14 where the neutral beam heating profile is dramatically altered but no change in the temperature profiles is observed. The power balance analysis there does not require non-diffusive transport, only a dramatic change in the diffusivity.

Electron cyclotron heating (ECH) experiments on DIII-D do provide direct evidence of non-diffusive transport $[15,16]$. Despite $80 \%$ of the input power being deposited outside of a normalized radius of 0.3 , the electron temperature profile remains very peaked (Fig. 4). Power balance analysis of off-axis ECH discharges shows that the heat flux in the electron fluid must be inward (in the direction of increasing $T$ ) at radii smaller than the heating location to support the measured temperature (Fig. 5). The error in determining the flux is quite small and the best diagnosed cases have negative flux to $3 \sigma$. Thermodynamic constraints require the diffusivities to be positive; the total matrix is not required to be symmetric, but only to have a positive determinant to satisfy the Second Law [17]. A negative flux would imply a negative diffusivity in the power balance analysis in violation of these thermodynamic constraints; therefore, the heat flux must have a significant non-diffusive component. The plasma works effectively as a heat engine - moving heat against the electron temperature gradient, transferring it to the ions, and exhausting it through transport on the ion fluid.

It has not been possible to determine from power balance analysis which driving terms are correlated with this inward flux. However, perturbative analysis of temperature modulations induced by periodically varying the input ECH power [18] indicates the heat flux must have the form

$$
q=-n \chi \nabla T+f T
$$

The function $f$ is often written as $n U$ where $U$ has units of velocity in analogy to the standard-particle balance equation. It is interesting that non-diffusive transport has now been observed in momentum transport experiments [19], indicating the ubiquitous nature of this phenomenon. 


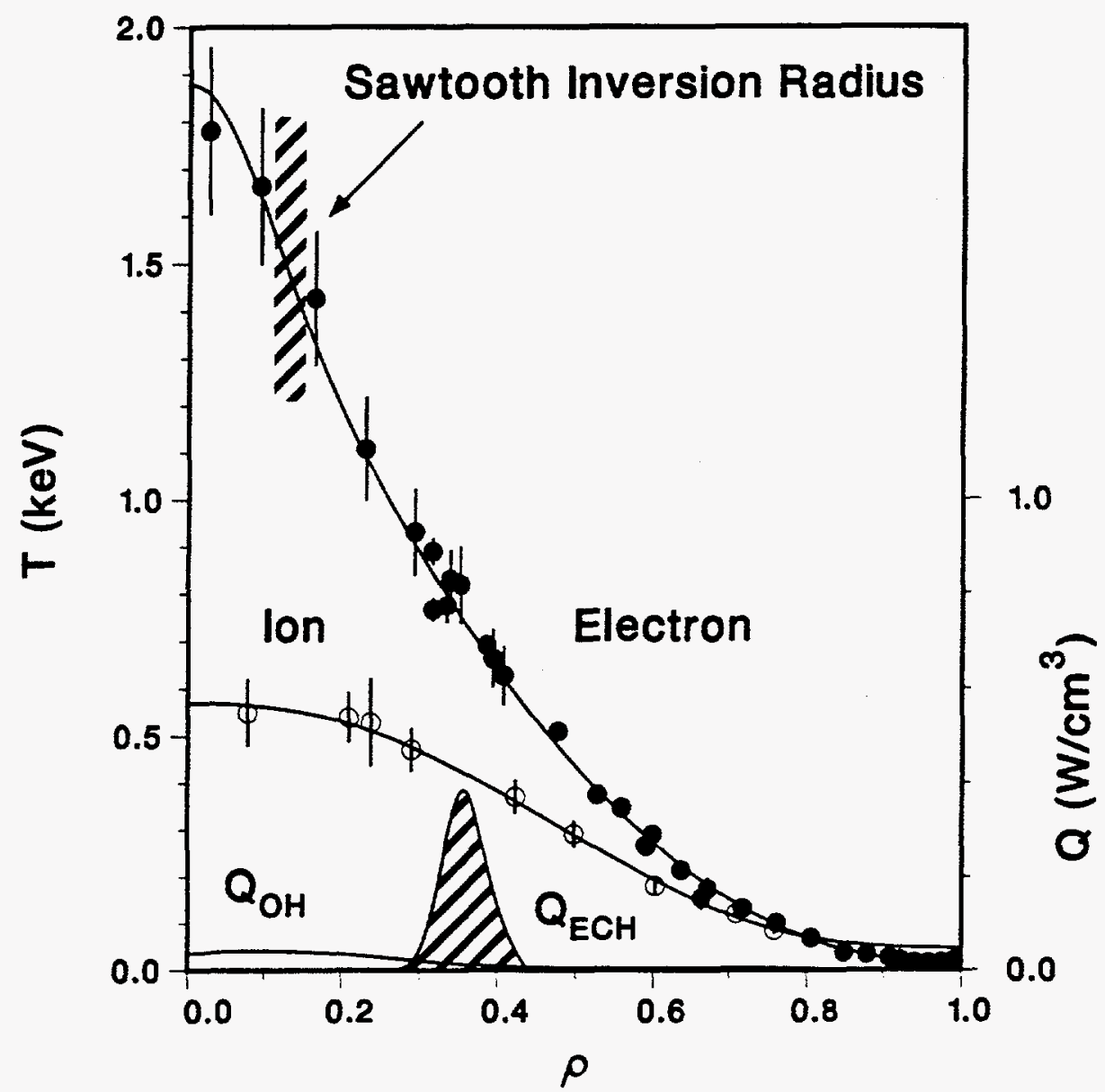

FIG. 4. Plot of electron and ion temperature measurements versus $\rho_{*}$. The electron temperature does not respond to the localized heating as expected for a diffusive system. The heating location is well outside the sawtooth mixing radius. The discharge parameters are $I=0.5 \mathrm{MA}, \bar{n}=2.8 \times 10^{13} \mathrm{~cm}^{-3}, B=1.8 \mathrm{~T}$, and $P_{\mathrm{ECH}}=0.85 \mathrm{MW}$.

Some progress has been made in reproducing this inward heat flux in quasilinear drift wave calculations. A fluid model which includes toroidal effects, impurities, and collisions in calculation of the transport matrix elements for particle, electron heat, and ion heat transport reproduces the inward flux in a self-consistent simulation $[20,21]$. This model has also been successful in reproducing TFTR [22] and JET [23] profiles. Although some parts of the model such as the lack of ion Landau damping are not universally accepted in the theoretical community, the broad success of the model should invite 


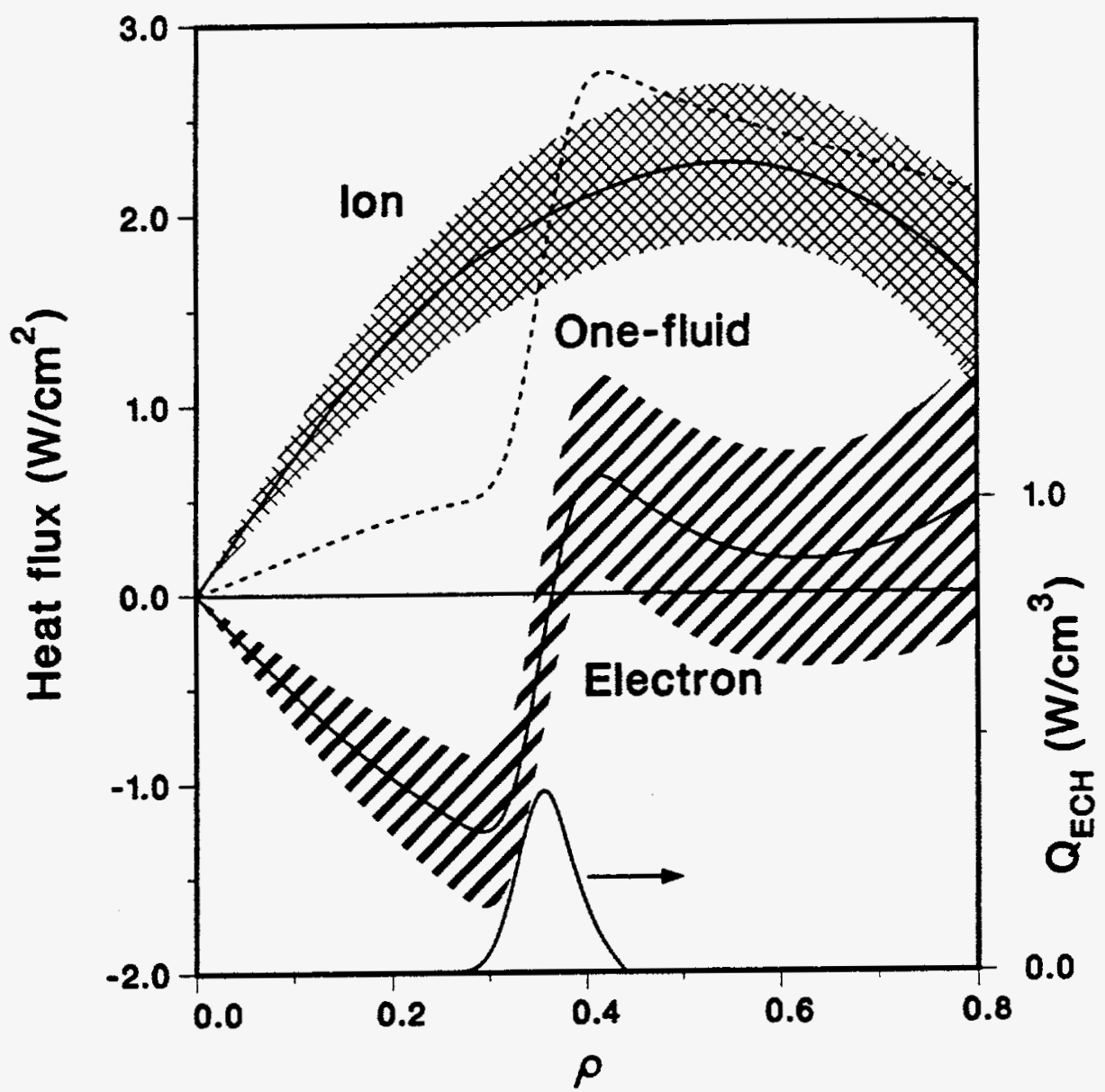

FIG. 5. Plot of ion, electron, and total heat flux versus $\rho_{*}$ for the discharge shown in Fig. 4. The total flux is positive everywhere as required by thermodynamics. Error bands are calculating the variation on the fluxes due to potentially systematic errors in the input data.

broader testing by the experimental community and more intense scrutiny by the theoretical community.

\section{EFFECT OF NON-DIFFUSIVE TERMS ON DIMENSIONLESS SCALING}

At first glance, the results of Section 3 may appear to invalidate the work of Section 2, since Eq. (1) explicitly characterizes transport as a diffusivity. However, Eq. (1) can be extended to include off-diagonal terms. For example, if the heat flux is 


$$
q=-n \chi \nabla T-T \chi^{*} \nabla n
$$

then the diffusivity can be written $\chi\left[1+(1 / \eta)\left(\chi^{*} / \chi\right)\right]$. Must $\chi^{*}=0$ to apply the concepts of dimensionless scaling? If $\chi$ is written as Eq. (1) and $\chi^{*}=\chi_{\mathrm{B}}\left(\rho_{*}\right)^{\gamma} G$, then the necessary condition for this effective diffusivity to be an appropriate scaling variable is that $\alpha=\gamma$; i.e., the $\rho_{*}$ scaling of both contributions to the flux must be the same.

Preliminary analysis of perturbative transport data from modulated ECH shows that the off-diagonal term does indeed have the same $\rho_{*}$ scaling as the diagonal term. For low frequency modulation on DIII-D, the off-diagonal term dominates the simple estimates of the diffusivity [18]. Using the asymptotic formula for the phase estimate of the diffusivity in the limit of low frequency [18], this measure of the transport should increase with the square of the actual diffusivity. In the case of the low density if dimensionless scaling pair, the global confinement time and the local power balance diffusivity scale in the same way (see Fig. 2). Using the ratio of the confinement times $R$ as a measure of the ratio of the true diffusivities, the low frequency phase estimates are found to vary as $R^{1.8}$. This is taken as preliminary evidence that the off-diagonal term scales with $\rho_{*}$ as the diagonal term.

\section{REFERENCES}

[1] “The ARIES-I Tokamak Reactor Study, Final Report 1991," UCLA-PPG1323.

[2] UCKAN, N.A., ITER Physics Group, "ITER Physics Design Guidelines: 1989," ITER Documentation Series, No. 10, IAEA, Vienna, 1990.

[3] KADOMTSEV, B.B., Sov. J. Plasma Phys. 1 (1975) 295.

[4] CONNOR, J.W., TAYLOR, J.B., Nucl. Fusion 17 (1977) 1047.

[5] GOLDSTON, R.J., Plasma Phys. and Contr. Fusion 26 (1984) 87.

[6] PETTY, C.C., et al., in Radio-Frequency Power in Plasmas 1993 (Proc. 10th Topical Conf. Boston) (AIP, New York, 1994) p. 165.

[7] STROTH, U., et al., Phys. Rev. Lett. 70 (1993) 936.

[8] CHRISTIANSEN, J.P., et al., Nucl. Fusion 33 (1993) 863.

[9] PERKINS, F.W., et al., Phys. Fluids B 5 (1993) 477.

[10] BURRELL, K.H., et al., in Plasma Physics and Controlled Nuclear Fusion Research 1990 (Proc. 13th Int. Conf. Washington, DC) (IAEA, Vienna, 1991), Vol. 1, p. 123.

[11] COPPI, B., SHARKY, N., Nucl. Fusion 21 (1981) 1363.

[12] COPPI, B., MAZZUCATO, E., Phys. Lett. 71 (1979) 337.

[13] DNESTROVSKY, Yu.N., et al., Sov. J. Plasma Phys. 16 (1990) 120.

[14] SCHISSEL, D.P., et al., Nucl. Fusion 32 (1992) 689,

[15] LUCE, T.C. et al., Phys. Rev. Lett. 68 (1992) 52.

[16] PETTY, C.C., LUCE, T.C., Nucl. Fusion 34 (1994) 121.

[17] BOOZER, A.H. Phys. Fluids B 4 (1992) 2845. 
[18] LUCE, T.C, et al., in Local Transport Studies in Fusion Plasmas (Società Italiana di Fisica, Bologna, 1993) p. 155.

[19] NAGASHIMA, K., et al., ibid., p. 75.

[20] NORDMAN, H., et al., Nucl. Fusion 30 (1990) 983; FRÖJDH, M., et al., Nucl. Fusion 32 (1992) 419; WEILAND, J., Chalmers University of Technology Report CTH-IEFT/PP-1993-23 (1993).

[21] WEILAND, J., NORDMAN, H., Phys. Fluids B 5 (1993) 1669.

[22] BATEMAN, G., et al., "Transport in Fusion Plasmas" (Chalmers University of Technology, Göteborg, 1994), to be published.

[23] CONNOR, J., et al., ibid. 